\title{
Modelado geoestadístico de fertilidad de un terreno agrícola mediante mapas de Kriging que interpolan los datos de análisis químico de suelos
}

\section{Carlos Roberto Martínez}

\author{
Maestro en Agronomía \\ Docente investigador \\ Facultad de Ingeniería y Arquitectura \\ Universidad Católica de El Salvador, El Salvador \\ email: carlos.martinez@catolica.edu.sv \\ ORCID: https://orcid.org/0000-0002-6098-8765
}

Recepción: 02/10/2019 Aceptación: 05/05/2020

\section{Resumen}

Dado que la fertilidad del suelo puede variar grandemente en distancias relativamente cortas, el método estadístico clásico no es adecuado para analizar los datos provenientes de análisis químicos de suelos.

El método de interpolación de Kriging, en cambio, es capaz de interpolar datos para generar, con cierto nivel de confianza, mapas georreferenciados de dichas propiedades. En este estudio, se realizaron 25 análisis de suelo pertenecientes a un mismo terreno para obtener datos de $\mathrm{pH}$, la capacidad efectiva de intercambio catiónico, disponibilidad de nutrientes y presencia de anti nutrientes; los cuales fueron tabulados junto con sus respectivas coordenadas.

El software Statgraphics 18 y PSPP exhibieron valores altos de desviación estándar, curtosis o de asimetría con respecto a la media de cada serie de datos, lo cual invalidó la confiabilidad de posibles regresiones y correlaciones. En cambio, los análisis de variograma hechos mediante Vesper ${ }^{\circledR}$ arrojaron datos de nugget y meseta que indicaron la existencia de correlación espacial, haciendo confiable el uso de la forma ordinaria de Kriging: " $a+b(x)$ ", para generar una capa raster de datos interpolados por cada variable, a través del software QGIS y SAGA, usando un tamaño de celda de una milésima para una buena resolución.

Esta función calcula cada interpolación como el promedio de los valores circundantes más su rezago espacial, sumando a continuación la varianza estimada entre los puntos circundantes. Con base al raster se trazaron curvas de contorno para delimitar las áreas con valores semejantes. Los mapas permitieron la identificación de las condiciones del terreno en función de la ubicación.

Palabras clave: Fertilidad de suelo, nutrición vegetal, Kriging, GIS, agricultura de precisión.

\begin{abstract}
Since soil fertility can vary greatly over relatively short distances, the classical statistical method is not suitable for analyzing data from chemical analyzes of soils.

The Kriging interpolation method, on the other hand, is capable of interpolating data to generate, with a certain level of confidence, georeferenced maps of these properties. In this study, 25 soil analyzes belonging to the same field were carried out to obtain data on $\mathrm{pH}$, the effective cation exchange capacity, availability of nutrients and the presence of anti-nutrients; which were tabulated together with their respective coordinates.

Statgraphics 18 and PSPP software exhibited high values of standard deviation, kurtosis, or skewness with respect to the mean of each data series, which invalidated the reliability of possible regressions and correlations. On the other hand, the variogram analysis made using Vesper ${ }^{\circledR}$, yielded nugget and plateau data that indicated the existence of spatial correlation, making reliable the use of the ordinary form of Kriging: " $a+b(x)$ ", to generate a layer raster of data interpolated by each variable, through QGIS and SAGA software, using a cell size of one thousandth for good resolution.

his function calculates each interpolation as the average of the surrounding values plus their spatial lag, then adding the estimated variance between the surrounding points. Based on the raster, contour curves were drawn to delimit the areas with similar values. Maps allowed the identification of land conditions based on location.
\end{abstract}

Key words: Soil fertility, vegetal nutrition, Kriging, GIS, precision farming. 


\section{Introducción}

Muchos son los factores que generan la disponibilidad de nutrientes en un campo de cultivos; y a menudo la relación entre ellos parece ser aleatoria. Pero en realidad, estos son producto de fenómenos naturales complejos o las prácticas agrícolas que se han realizado con anterioridad. Entre los factores que pueden causar tal variabilidad puede mencionarse la ocurrencia de escorrentías (resultado típico de alterar campiña salvadoreña), donde el pH desciende, debido a la pérdida de minerales como el calcio (Achudume, 2007; Kato et al., 1996; Qureshi et al., 2016).

A consecuencia, cuando los suelos son demasiado ácidos, el aluminio (Al) se vuelve disponible y provoca reacciones tipo redox, que interfieren con la absorción de nutrientes como el fosfato (Pant et al., 2002; Scanlan et al., 2017). Otro fenómeno importante es que los niveles de capacidad efectiva de intercambio catiónico (ECEC, por sus siglas en inglés $)^{1}$; también tienden a manifestar una variabilidad espacial considerable por muchas razones, tales como actividad microbiana, diferencias en el manejo de micro parcelas, inundaciones, escorrentías y contenido de materia orgánica (Grant, 2018; Liang et al., 2006; Schalscha et al., 1974; Hendershot et al., 1993).
El impacto que estos complejos fenómenos ejercen sobre el suelo puede modelarse, mediante la creación de bases de datos que puedan importarse en un software de información geográfica (GIS, por sus siglas en inglés), el cual puede -a su vez- generar mapas visuales para identificar los requerimientos específicos de sitio, ayudando en un proceso de toma de decisiones que coopera con el uso eficiente de fertilizantes químicos (Denton et al, 2017; Rogowski, 1996). Tales modelos pueden también usarse con interés predictivo, ya que hacen posible que al tomar muestras georeferenciadas de suelo, estas últimas puedan ser sujeto de diferentes tratamientos en laboratorio, posibilitando recabar nuevos datos y crear un modelo representativo de respuesta en campo, sin incurrir en altos costos ni en labores exhaustivas.

Este artículo discute acerca de un caso real de uso de herramientas de software para la creación mapas de interpolación de Kriging, y las aplicaciones que pueden tener desde el punto de la planeación para manejo eficiente de cultivos agronómicos.

El algoritmo ordinario de Kriging asume que los valores medios y de varianza de una serie de datos espaciales (bidimensionales) son constantes, a través de la distancia siempre; y que los valores no cambian drásticamente

1. El autor también se referirá a este término mediante sus siglas. 
en función del tiempo, tal como sucede con el suelo. El algoritmo ordinario de Kriging expresa cada valor muestral en función de su posición: $z(x)$.

La suma ponderada de los valores de muestras circundantes se denomina rezago espacial " $h$ " (Gia et al., 2019). La media general del valor muestral “ $m$ ” más su respectivo rezago es $z(x+h)$, por lo cual la ecuación se escribe como $m=z(x+h)$. Al sustituir la expresión de la media y adicionar la función espacial de la varianza $g(x)$, su ecuación resultante es: $E(x)$ $=m+g(x)($ Negreiros et al., 2010), con la cual puede cuantificarse la variabilidad de muestras cercanas entre sí (Camana \& Deutsch, 2019). Si bien desde un punto de vista práctico, la deducción de este modelo matemático no es un requisito directo para los estudios de fertilidad del suelo, a nivel científico se utiliza para justificar la metodología y conocer el origen de los parámetros que se introducen para calibrar en el modelo de procesamiento de datos, mediante software, tal y como se muestra en el presente estudio.

La interpolación de Kriging ha resultado adecuada para comprender el fenómeno de fertilidad de suelos, a partir de 16 muestras vecinas con poca evidencia de anisotropía (Kabir et al., 2019); logrando establecer patrones de variabilidad progresiva y no aleatoria a través del terreno. Con esto se posibilita categorizar micro zonas de cultivo, incrementando la eficiencia del manejo agronómico con base en las propiedades modeladas de suelo, tal como en el caso de la fertilización específica de sitio basada en la disponibilidad de nutrientes (Farooque et al., 2012; Houlong et al., 2016; Jafarnejadi et al., 2013).

Por estas razones, los mapas de Kriging son reconocidos a nivel mundial como herramientas fiables y modernas para apoyar la toma de decisiones, y operar aplicaciones de agricultura de precisión (Panday et al., 2018), por lo cual, esta investigación discute dicha temática dentro de la realidad salvadoreña. Esto podría resultar de gran relevancia en un futuro próximo, dado que los recientes avances en tecnologías de sensores permiten recabar grandes cantidades de datos, y convertirlos en mapas agrícolas comercialmente viables para su adopción a gran escala (Nawar et al., 2017).

\section{Materiales y Métodos}

El terreno agrícola estudiado es parte del campo experimental de la Universidad Católica de El Salvador, cuya área aproximada es de 25 hectáreas de suelo pesado y tipo franco arcilloso. Tiene una pendiente máxima de $39.3 \%$ y un promedio de $18.7 \%$. En él se han establecido durante décadas cultivos tradicionales de El Salvador por parte de diferentes agricultores que, a su vez, ejecutaban distintas prácticas agrícolas; lo cual hacía posible asumir que las condiciones de fertilidad del suelo no eran uniformes. 
Se tomaron 25 muestras de suelo en concordancia con la proporción de una muestra por hectárea, recomendada por Amaral et al. (2019). El método de muestreo correspondió a un patrón de cuadrícula de cinco transectos paralelos, de acuerdo con la curvatura de la pendiente, según lo indicado por Gregorich y Carter (2007). En forma similar a las metodologías utilizadas por Walvoort et al. (2010), Ching et al. (2009), Cockx et al. (2007) y Fox y Protz (1981), cada muestra estuvo compuesta por 10 submuestras, tomadas en un patrón circular de 20 metros de diámetro. Luego se procedió a la captura de coordenadas GPS y etiquetado.

Las muestras de suelo fueron enviadas a un laboratorio certificado para determinar su $\mathrm{pH}$, ECEC expresado en Meq/100cc; porcentaje de materia orgánica ( $\mathrm{MO}$ ) y las concentraciones en PPM de fósforo $(\mathrm{P})$, potasio $(\mathrm{K})$, calcio (Ca), magnesio (Mg), azufre (S), hierro (Fe), zinc $(\mathrm{Zn})$, manganeso $(\mathrm{Mn})$, boro (B) y cobre $(\mathrm{Cu})$. Se midió, además, la concentración de aluminio $(\mathrm{Al})$ y sodio $(\mathrm{Na})$ para considerar su potencial antagónico. Los análisis químicos fueron llevados a cabo por un laboratorio privado acreditado, bajo la versión 2017 de la norma ISO/IEC $17025^{2}$.

Los datos obtenidos de las variables de cada muestra fueron tabulados y analizados con los software Statgraphics ${ }^{\bowtie} 18$ y GNU/PSPP 1.2, para obtener la estadística descriptiva y relación entre variables. Se calcularon los promedios de cada variable, su respectiva desviación estándar, los valores de curtosis y asimetría para analizar la forma de distribución de los datos, y así determinar la conveniencia del análisis $\mathrm{R}^{2}$ como método para la predicción de respuesta de una variable dependiente, con respecto a otra independiente.

Los datos fueron analizados mediante la creación de semivariogramas, mediante el software Vesper ${ }^{\circledast}$ (Bernardi et al. 2016; Molin y Faulin, 2013; Whelan et al., 2002), utilizando como parámetros un número de 30 lags $^{3}$, una tolerancia de lag del 50\% y una distancia máxima de cluster de 100 metros. Esto permitió calcular los valores de nugget y meseta, conocidos en la literatura como $\mathrm{c}_{0}, \mathrm{y} \mathrm{c}_{1}$, respectivamente. El software pudo determinar si había convergencia de los variogramas e identificar el tipo de modelo: esférico, exponencial, gaussiano o linear. Esto fue requisito para proceder confiablemente con el análisis de Kriging.

Los datos de cada punto muestral se organizaron en un archivo tipo CSV, que permitió importar la información como una capa de datos en el software QGIS 3.4. Mediante el plugin SAGA 7.3, se crearon mapas de varia-

2. Con la finalidad de agilizar la lectura, el autor utilizará los símbolos químicos para referirse a estos componentes.

3. Este término es utilizado en el ámbito tecnológico, específicamente en telecomunicación, para hacer referencia al retardo excesivo dentro del envío y recepción de datos. 
bilidad de Kriging usando el modelo ordinario, asumiendo que la media y la varianza de todos los valores era constante a través de todo el campo espacial, dada la escasa "movilidad" de las variables en estudio; autores como Pandey et al. (2009), Hlaing et al. (2008), Erdogan et al. (2007), Scull et al. (2003) y Zhu et al. (2001), siguieron similares metodologías.

La fórmula de Kriging ordinario, descrita previamente como $E(x)=m+g(x)$, fue introducida en el plugin de SAGA 7.3, anteriormente integrado en QGIS, representándola de la siguiente manera:

$$
E(x)=a+b(x)
$$

En donde:

El valor medio “ $m$ ” se expresó como parámetro " $a$ " y la varianza " $g$ " como parámetro " $b$ "; por lo que la ecuación del modelo ordinario tomó la forma $E(x)=a+b(x)$, y fue configurada en el software como simplemente: $a+b^{\star} x$.

Para obtener una buena resolución, el tamaño de celda interpolada se fijó en la escala de una milésima y el tamaño de bloque a 100. Este fue el procedimiento usado para generar cada una de las capas raster, una por cada variable química del archivo CSV. Posteriormente, se utilizó la herramienta de Contornos de SAGA para crear otra capa con áreas delimitadas, que permitiera agregar texto y color personalizado para el mejoramiento visual. Finalmente, se usaron los mapas de Kriging para identificar las zonas con mayor y menor $\mathrm{pH}, \mathrm{ECEC}, \mathrm{Al}$ y $\mathrm{Na}$; obteniendo sus respectivos valores promedio de $\mathrm{Ca}, \mathrm{Mg}, \mathrm{K}, \mathrm{P}$ y Fe para calcular las relaciones $\mathrm{Ca} / \mathrm{Mg}, \mathrm{Ca} / \mathrm{K}$, (Ca-Mg)/K, Mg/K, Ca/P y Fe/P, con el fin de considerarlos como indicadores de fertilidad (Matos et al., 2017; Shi et al., 2017).

\section{Resultados y discusión}

Las tablas 1 y 2 muestran los datos obtenidos de los análisis químicos de suelo. Se pudo observar poca representatividad de la media, causada por la variación de las condiciones dentro de la misma propiedad. La mayoría de muestras presentaron valores bajos de materia orgánica (ECEC, pH, $\mathrm{P}$ y K), lo cual es típico de terrenos con un serio decaimiento de fertilidad.

Pudo notarse grandes variaciones en la disponibilidad del calcio, pero aún en las zonas con concentraciones "suficientes", el pH se mantuvo muy bajo y la presencia de otros nutrientes era limitada, debido a la pobre ECEC.

La tabla 2 detalla las altas concentraciones de $\mathrm{Al} \mathrm{y} \mathrm{Na}$, versus la baja disponibilidad de micronutrientes, demostrando que el desbalance en la fertilidad del suelo induce, simultáneamente, a situaciones de severas deficiencias y a otras de leve toxicidad. 
Tabla 1. Datos de posición, indicadores de fertilidad y disponibilidad de macro y micronutrientes

\begin{tabular}{|c|c|c|c|c|c|c|c|c|c|c|}
\hline Sitio & Latitud $^{(1)}$ & Longitud $^{(2)}$ & $\mathrm{pH}$ & $\begin{array}{c}\text { Porcentaje } \\
\text { de MO }\end{array}$ & $\begin{array}{c}\text { ECEC } \\
(m E q / 100 c c)\end{array}$ & $\mathbf{P}^{*}$ & $\mathbf{K}^{*}$ & $\mathrm{Ca}^{*}$ & $\mathbf{M g}^{*}$ & $S^{*}$ \\
\hline 1 & 14.023322 & -89.659357 & 4.5 & $2.3 \%$ & 9 & 5 & 75 & 905 & 355 & 56.7 \\
\hline 2 & 14.022043 & -89.659333 & 4.1 & $3.74 \%$ & 6 & 6 & 108 & 665 & 200 & 79.2 \\
\hline 3 & 14.021367 & -89.659684 & 4.7 & $2.33 \%$ & 10 & 6 & 30 & 1180 & 305 & 40.1 \\
\hline 4 & 14.020407 & -89.659888 & 4.6 & $3.32 \%$ & 9 & 5 & 126 & 1050 & 260 & 33.5 \\
\hline 5 & 14.019383 & -89.659766 & 4.5 & $3.55 \%$ & 7 & 7 & 136 & 805 & 240 & 52.2 \\
\hline 6 & 14.023261 & -89.658506 & 4.4 & $2.88 \%$ & 8 & 20 & 142 & 920 & 245 & 45 \\
\hline 7 & 14.022049 & -89.658666 & 4.4 & $3.19 \%$ & 7 & 7 & 800 & 215 & 215 & 35 \\
\hline 8 & 14.020769 & -89.658627 & 4.4 & $3.32 \%$ & 8 & 8 & 157 & 860 & 230 & 50.3 \\
\hline 9 & 14.020287 & -89.658661 & 4.4 & $3.82 \%$ & 6 & 8 & 86 & 630 & 210 & 53.1 \\
\hline 10 & 14.019535 & -89.658965 & 4.4 & $4.02 \%$ & 8 & 8 & 114 & 1035 & 235 & 54.3 \\
\hline 11 & 14.023517 & -89.657992 & 4.7 & $4.95 \%$ & 3 & 6 & 78 & 213 & 58 & 40.4 \\
\hline 12 & 14.020841 & -89.657996 & 4.4 & $4.86 \%$ & 9 & 23 & 106 & 985 & 270 & 57.6 \\
\hline 13 & 14.021374 & -89.658051 & 4.6 & $3.01 \%$ & 9 & 47 & 57 & 1085 & 240 & 74.6 \\
\hline 14 & 14.020147 & -89.658111 & 5.1 & $3.34 \%$ & 8 & 9 & 114 & 950 & 260 & 45.6 \\
\hline 15 & 14.019217 & -89.658438 & 4.4 & $4.89 \%$ & 8 & 22 & 96 & 835 & 210 & 36.3 \\
\hline 16 & 14.022742 & -89.657304 & 4.5 & $2.51 \%$ & 16 & 17 & 54 & 2215 & 420 & 39.3 \\
\hline 17 & 14.021581 & -89.657527 & 5.5 & $3.82 \%$ & 9 & 4 & 122 & 1125 & 275 & 28.7 \\
\hline 18 & 14.020596 & -89.657652 & 5.3 & $2.52 \%$ & 7 & 7 & 189 & 855 & 150 & 29.4 \\
\hline 19 & 14.019742 & -89.657624 & 6.1 & $5.73 \%$ & 14 & 5 & 178 & 1970 & 380 & 28.1 \\
\hline 20 & 14.018914 & -89.657707 & 4.4 & $2.98 \%$ & 7 & 11 & 92 & 945 & 180 & 35.7 \\
\hline 21 & 14.022888 & -89.655136 & 5.1 & $4.19 \%$ & 11 & 18 & 100 & 1480 & 295 & 30.8 \\
\hline 22 & 14.022118 & -89.655278 & 5.1 & $2.91 \%$ & 13 & 24 & 146 & 1695 & 385 & 29.6 \\
\hline 23 & 14.020582 & -89.655603 & 4.1 & $2.37 \%$ & 8 & 10 & 94 & 1000 & 275 & 38.7 \\
\hline 24 & 14.019802 & -89.656119 & 4.2 & $3.21 \%$ & 5 & 17 & 92 & 505 & 95 & 27.2 \\
\hline 25 & 14.018626 & -89.656671 & 4.8 & $3.36 \%$ & 8 & 22 & 88 & 1040 & 145 & 29.4 \\
\hline
\end{tabular}

Nota: ${ }^{(1)} \mathrm{y}^{(2)}$ coordenadas expresadas en formato decimal, latitud Norte y longitud Este. // * expresados en ppm. 
Tabla 2. Disponibilidad de micro y antinutrientes

\begin{tabular}{|c|c|c|c|c|c|c|c|}
\hline Sitio & $\mathrm{Cu}^{*}$ & $\mathrm{Fe}^{*}$ & $\mathrm{Mn}^{*}$ & $\mathrm{Zn}^{*}$ & $\mathbf{B}^{*}$ & $\mathbf{A l}^{*}$ & $\mathrm{Na}^{*}$ \\
\hline 1 & 4.1 & 48.3 & 86 & 3 & 1.7 & 90 & 102.5 \\
\hline 2 & 5.6 & 40.8 & 107 & 3.9 & 0.8 & 99 & 108.1 \\
\hline 3 & 4.3 & 39.8 & 86 & 3.7 & 1 & 90 & 68.1 \\
\hline 4 & 4.1 & 37.9 & 109.5 & 3 & 0.4 & 90 & 88.8 \\
\hline 5 & 4.3 & 40.9 & 87 & 15.7 & 0.2 & 99 & 95.6 \\
\hline 6 & 3 & 42.1 & 91.5 & 2 & 0.8 & 117 & 63.1 \\
\hline 7 & 3.4 & 44.4 & 75 & 4.2 & 0.6 & 117 & 61.9 \\
\hline 8 & 4.3 & 48.5 & 90.5 & 1.6 & 0.8 & 126 & 108.1 \\
\hline 9 & 4.4 & 35.7 & 88 & 1.8 & 1.7 & 108 & 57.4 \\
\hline 10 & 4.3 & 36.4 & 97.5 & 2.6 & 0 & 99 & 58.1 \\
\hline 11 & 2.4 & 47.9 & 58.5 & 0.8 & 1.8 & 90 & 88.1 \\
\hline 12 & 1.8 & 32.8 & 69.5 & 2.2 & 2.4 & 126 & 50 \\
\hline 13 & 2.9 & 49.6 & 96.5 & 6 & 0.9 & 90 & 70 \\
\hline 14 & 2.9 & 48.6 & 45.8 & 5 & 0.9 & 81 & 66.3 \\
\hline 15 & 2.2 & 38.3 & 97.5 & 2.3 & 1 & 153 & 108.8 \\
\hline 16 & 1.2 & 20.6 & 74 & 1.9 & 1 & 99 & 108.1 \\
\hline 17 & 2.3 & 25.9 & 59.5 & 1.7 & 0.9 & 90 & 106.9 \\
\hline 18 & U.Y & 19.9 & 53 & $b$ & U. & yu & 84.4 \\
\hline 19 & 0.7 & 7.2 & 48 & 2.8 & 0.9 & 63 & 113.1 \\
\hline 20 & 1.6 & 30.7 & 86 & 3.2 & 1.2 & 90 & 70.6 \\
\hline 21 & 2.6 & 19.8 & 54.5 & 3.6 & 0.8 & 72 & 71.9 \\
\hline 22 & 2.6 & 24.1 & 105.1 & 8.2 & 0.7 & 72 & 53.1 \\
\hline 23 & 1.6 & 29 & 64 & 6.2 & 0.9 & 90 & 62.5 \\
\hline 24 & 1.2 & 46.5 & 61 & 3.6 & 0.6 & 99 & 81.9 \\
\hline 25 & 2.8 & 21.7 & 80.5 & 9.1 & 0.8 & 99 & 58.1 \\
\hline
\end{tabular}

Nota: ${ }^{\star}$ expresado en ppm. 
Análisis estadístico

La amplia diferencia entre los valores máximos y mínimos de casi todas las propiedades y nutrientes, condujo a valores altos de desviación estándar; y comprobó que la información espacial no era uniforme ni fácilmente predecible (ver tabla 3 ). El nutriente con mayor inconsistencia fue el potasio, dado que el valor de la desviación estándar es incluso mayor al del promedio. Los valores de curtosis y asimetría indicaron la ausencia de normalidad, lo cual determinó como improcedente cualquier análisis de regresión o correlación de variables.

En un sentido práctico, quedó claro que el análisis estadístico tradicional es demasiado limitado para describir el fenómeno de variabilidad espacial. Con base a lo observado, implementar estrategias de manejo agronómico que sean uniformes para todas las micro parcelas, sería la peor de las opciones en términos de eficacia de la inversión y producción de cosechas (ver tabla 3).

\section{Convergencia de variogramas}

Los datos de disponibilidad de boro y aluminio convergieron con un modelo lineal, ya que las varianzas entre los datos mostraron un valor de rango dentro de su respectivo promedio (aplanamiento de la curva o meseta); aunque con cierta discontinuidad, con respecto al origen debido a la poca variabilidad entre los datos de puntos cercanos.
El sodio también mostró una tendencia lineal debido a su claro comportamiento de meseta, pero con una muy alta variabilidad inicial entre datos cercanos al origen; por lo cual, el software lo declaró no convergente con algún modelo específico, pese a que existía correlación entre varianzas según lo indicó el valor de meseta (ver tabla 4).

Los valores de ECEC y P convergieron con un modelo gaussiano, debido a que los cálculos de las variaciones tenían un comportamiento parabólico, sin alcanzar nunca un valor estable de meseta. Sin embargo, tuvieron un comportamiento de correlación de varianzas en función de la distancia.

En el caso del K, Ca, Mg, Cu y Fe hubo convergencia con el modelo esférico, ya que todas las variables tuvieron una progresión lineal desde el punto de nugget, demostrando poca variabilidad entre cortas distancias, pero con una meseta bien definida en distancias largas.

El pH, el porcentaje de MO y las concentraciones de S, Mn y Zn, convergieron con el modelo exponencial, debido a que alcanzaban el rango en forma asintótica en función de la distancia. Así como en el modelo esférico, las variaciones son de carácter lineal a cortas distancias; pero se elevan abruptamente en función de la distancia y tienen una meseta lejana al origen. 
Tabla 3. Estadísticas descriptivas

\begin{tabular}{|c|c|c|c|c|c|c|}
\cline { 2 - 7 } \multicolumn{1}{c|}{} & Media & $\begin{array}{c}\text { Desviación } \\
\text { Estándar }\end{array}$ & Curtosis & Asimetría & Mínimo & Máximo \\
\hline $\mathrm{pH}$ & 4.7 & 0.5 & 2.4 & 1.5 & 4.1 & 6.1 \\
\hline $\mathrm{M} . \mathrm{O}$. & 3.5 & 0.9 & 0.3 & 0.8 & 2.3 & 5.7 \\
\hline $\mathrm{ECEC}$ & 8.5 & 2.8 & 1.8 & 0.9 & 3.0 & 16.0 \\
\hline $\mathrm{P}$ & 12.9 & 9.7 & 5.1 & 2.0 & 4.0 & 47.0 \\
\hline $\mathrm{K}$ & 135.2 & 143.4 & 21.3 & 4.5 & 30.0 & 800.0 \\
\hline $\mathrm{Ca}$ & 1006.5 & 458.6 & 1.6 & 0.9 & 213.0 & 2215.0 \\
\hline $\mathrm{Mg}$ & 245.3 & 85.6 & 0.3 & 0.0 & 58.0 & 420.0 \\
\hline $\mathrm{S}$ & 42.8 & 14.1 & 0.9 & 1.1 & 27.2 & 79.2 \\
\hline $\mathrm{Cu}$ & 2.9 & 1.3 & -0.8 & 0.2 & 0.7 & 5.6 \\
\hline $\mathrm{Fe}$ & 35.1 & 11.5 & -0.3 & -0.6 & 7.2 & 49.6 \\
\hline $\mathrm{Mn}$ & 78.9 & 19.1 & -1.1 & -0.2 & 45.8 & 109.5 \\
\hline $\mathrm{Zn}$ & 4.1 & 3.2 & 7.0 & 2.4 & 0.8 & 15.7 \\
\hline $\mathrm{B}$ & 0.9 & 0.5 & 2.1 & 1.0 & 0.0 & 2.4 \\
\hline $\mathrm{Al}$ & 97.6 & 19.2 & 1.9 & 1.0 & 63.0 & 153.0 \\
\hline $\mathrm{Na}$ & 80.2 & 20.9 & -1.5 & 0.3 & 50.0 & 113.1 \\
\hline
\end{tabular}

La tabla 4 muestra en detalle los coeficientes calculados por el análisis de variograma para cada variable. Pese que todos los modelos eran convergentes, a excepción del sodio, es probable que una mayor cantidad de muestras hubiese podido incrementar la confiabilidad de Kriging, tal como en el caso del fósforo, azufre, hierro, manganeso y aluminio; ya que el nugget $c_{0}$ fue mayor que la meseta $c_{1}$, lo cual demuestra que los datos están más relacionados en largas distancias que en el vecindario de cada punto. Esta afirmación está en total acuerdo con el hecho estadístico conocido, que la media puede ser calculada con mayor confiabilidad que el valor de un punto específico.

En el caso del pH, Ca, B y MO, los valores $\mathrm{c}_{0}$ fueron iguales o muy similares a $c_{1}$, lo cual es una manifestación del "efecto puro de nugget" que sucede cuando los datos no varían en función de la distancia; por lo cual Kriging otorga el mismo "peso" a todos los puntos. Esto le restó relevancia al centro de la muestra para dárselo a los puntos periféricos; situación que, aunque no es inusual, puede 
Tabla 4. Datos de ajuste de variograma

\begin{tabular}{|c|c|c|}
\hline Variable & $\boldsymbol{c}$ & $\boldsymbol{c} 1$ \\
\hline $\mathrm{pH}$ & 0.00 & 0.18 \\
\hline $\mathrm{MO}$ & 0.37 & 0.37 \\
\hline $\mathrm{CICE}$ & 2.89 & 7.10 \\
\hline $\mathrm{P}$ & 63.62 & 0.00 \\
\hline $\mathrm{K}$ & 112.60 & 1187.6 \\
\hline $\mathrm{Ca}$ & 5000.00 & 5000.00 \\
\hline $\mathrm{Mg}$ & 1595.10 & 5000.00 \\
\hline $\mathrm{S}$ & 156.20 & 27.08 \\
\hline $\mathrm{Cu}$ & 0.37 & 1.37 \\
\hline $\mathrm{Fe}$ & 104.40 & 9.09 \\
\hline $\mathrm{Mn}$ & 311.90 & 23.93 \\
\hline $\mathrm{Zn}$ & 0.00 & 8.17 \\
\hline $\mathrm{B}$ & 0.2499 & 0.00 \\
\hline $\mathrm{Al}$ & 316.1 & 0.00 \\
\hline $\mathrm{Na}$ & 37.44 & 216.3 \\
\hline
\end{tabular}

disminuir hasta cierto punto la confianza de complejas, ya que constituyó de un conjunto las interpolaciones en vecindarios cercanos a de datos multidimensionales en el cual cada cada punto.

$\mathrm{El} \mathrm{K,} \mathrm{Mg,} \mathrm{Cu,} \mathrm{Zn,} \mathrm{y} \mathrm{Na} \mathrm{manifestaron} \mathrm{un} \mathrm{valor}$ variable tipo "muestra" tenía su propio subconjunto de variables de tipo "propiedad", co mayor a $c_{1}$, por lo que se asume cierta confiabilidad de los variogramas, ya que la variabilidad se incrementó proporcionalmente en "nutriente" o "antinutriente". En la figura 1 se representa la topografía del terreno y la distribución de los puntos de muestreo. función de la distancia.

El método de Kriging generó planos de interpolaciones basadas en cada variable, en

\section{Creación del modelo GIS}

La base de datos que se desarrolló a partir de forma transversal a todas las muestras. Para ello hay que considerar que los datos no se vinculan únicamente a las muestras vecinas, los análisis de suelos, conllevaba relaciones 
sino que dependen de los valores obtenidos de otras muestras, a partir de las cuales se calcula una matriz de posibilidades que se representa como una capa raster, sobrepuesta en un sistema de coordenadas administrado por el software GIS.

Si bien la exactitud del modelo puede incrementarse agregando más puntos muestrales, eso también implica aumentar el tiempo de duración y los costos de un estudio; motivo por el cual se recomienda un análisis previo de conveniencia para cada terreno que haya necesidad de estudiar, considerando al mis- mo tiempo que el uso de muy pocas muestras puede afectar la confiabilidad del algoritmo de Kriging.

La figura 1 muestra un esquema de las curvas a nivel del terreno; mientras que la figura 2, por su parte, muestra el proceso de transformación de cada uno de los productos resultantes; partiendo de una capa raster de datos interpolados, sobre la cual se sobrepuso otra capa con líneas de contorno calculadas, con base a la agrupación de las zonas con datos semejantes. A esto se le suministraron mejoras en su presentación estética.

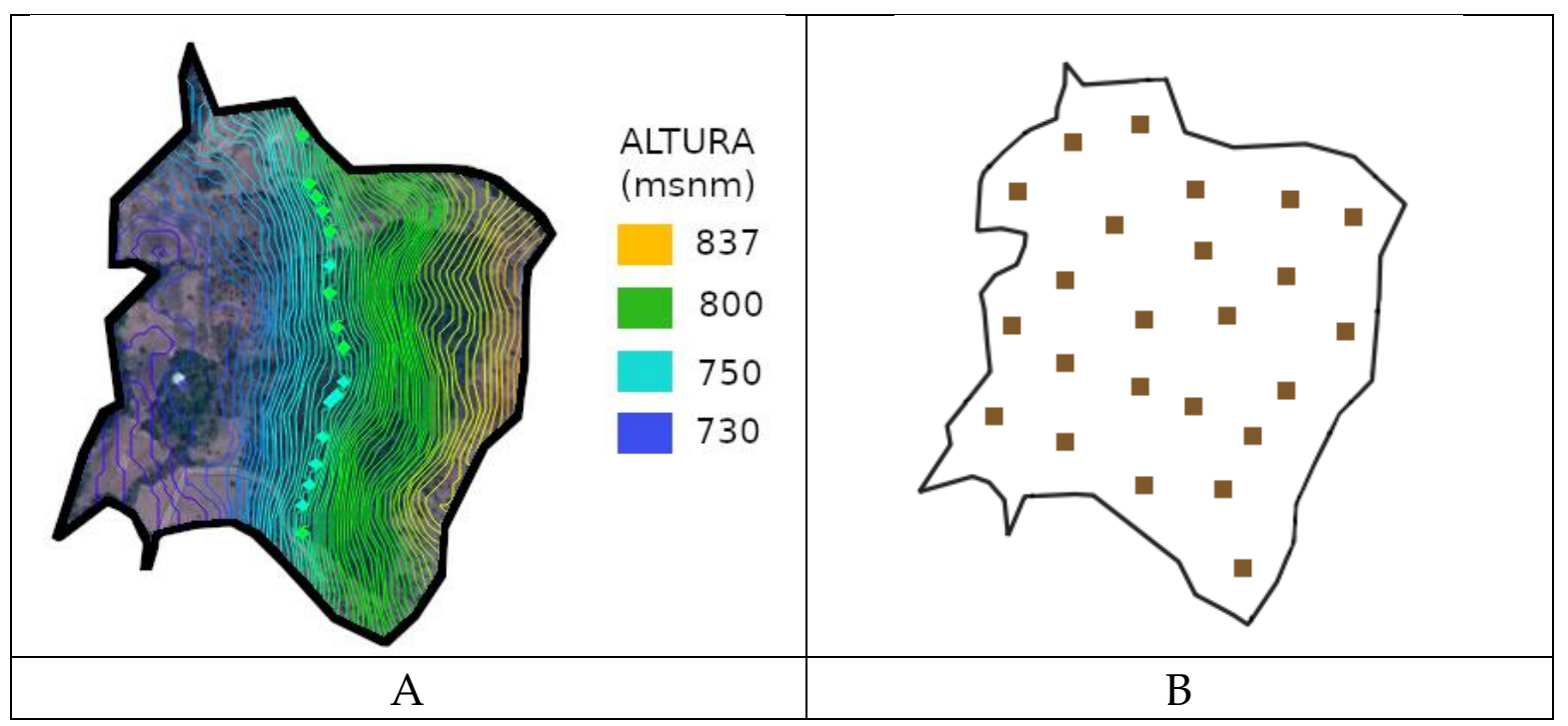

Figura 1. Representación de la topografía del terreno (A) y de la distribución de los puntos de muestreo (B). 


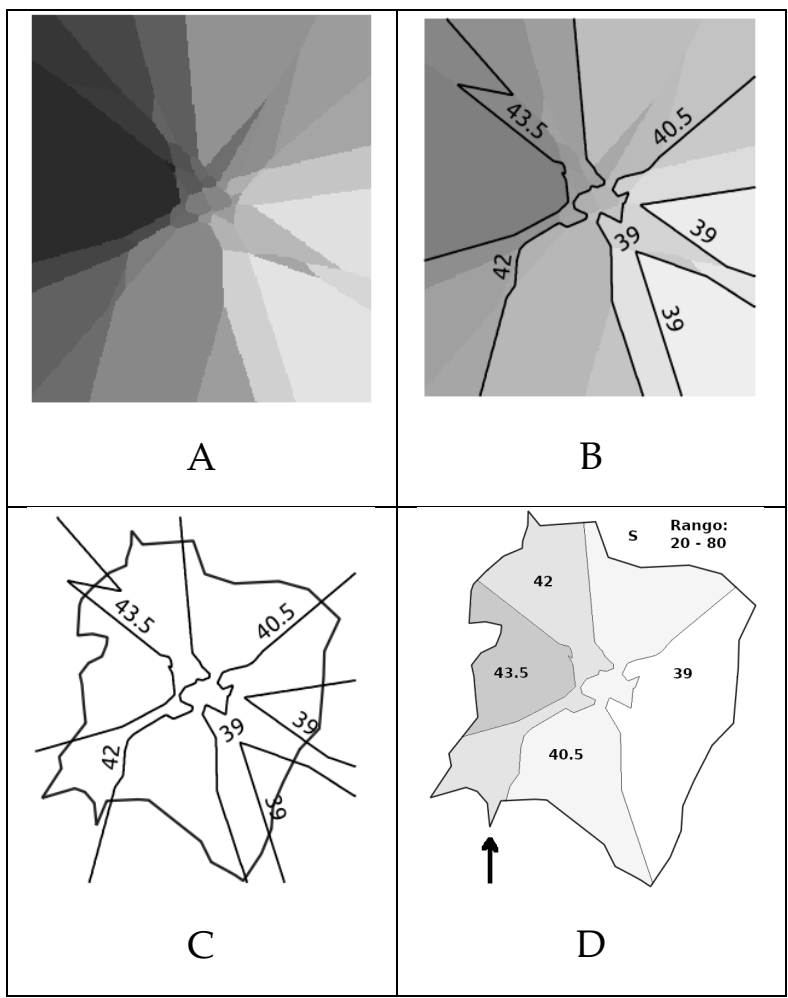

Figura 2. Pasos sugeridos para crear mapas de fertilidad, luego de cargar los puntos de datos, que son los mismos puntos de muestreo: (A) interpolar la capa raster mediante Kriging; (B) superposición de líneas de contorno con etiquetas; $(C)$ incorporación de perímetro del terreno y $(D)$ mejora estética. Los valores mostrados son de PPM disponible de azufre y su rango normal. La flecha en D apunta hacia las coordenadas de referencia (14.018608,-89.659766).

\section{Mapas GIS producidos}

El conjunto de mapas de Kriging de todas las variables se consideró como el perfil de fertilidad de suelo (figuras 4 y 5), el cual pudo representar desde pequeñas a grandes variaciones espaciales. Mediante tales mapas, se volvió fácil identificar las verdaderas condiciones a nivel de micro parcelas. Por ejemplo, las áreas con menor disponibilidad de $\mathrm{P}$, mostraron mayor acidez y alta presencia de $\mathrm{Al}$; por lo cual se dedujo que para su tratamiento es necesario encalar.
Por otra parte, todo el terreno debe considerarse como pobre en $\mathrm{Zn}$ y $\mathrm{Cu}$, pero solo una parte debe considerarse baja en B. La topografía del terreno influyó moderadamente en los resultados, probablemente por la variabilidad que agregó el manejo desigual de las múltiples microparcelas que se trabajaron internamente durante décadas. Las variables que demostraron tener vinculación con la topografía por erosión y deposición de partículas fueron el pH, Al, Na, S, Fe, y Mn. Otras variables como el $\mathrm{P}, \mathrm{K}$ y $\mathrm{Zn}$ mostraron un efecto 
inverso, probablemente por la reducción de elementos anti nutricionales ( $\mathrm{Al}$ y $\mathrm{Na})$.

La acumulación de nutrientes por escorrentías fue verificable en zonas donde el suelo era ligeramente más ácido, y por lo cual hubo una mayor disponibilidad en lugar de incrementar la deficiencia. En el caso de ECEC, $\mathrm{K}$ y Zn se observó que sus valores eran ligeramente más altos en función de una mayor presencia de MO.

Por otra parte, una vez interpolados los datos, la media ponderada de cada zona que se delimitó en los mapas no era tan dispar como lo anticipaba el análisis de estadística descriptiva, mostrando en su lugar una variabilidad espacial moderada. Eso se debe a que el proceso de interpolación reduce el "efecto de ruido", causado por algún dato "defectuoso", originado por el error humano o algunas condiciones alteradas en el lugar de la muestra. Esto mejora la calidad de los resultados y proporciona información más valiosa para la toma de decisiones (ver figuras 3 y 4 ).

La tabla 5 muestra las relaciones importantes entre cationes del suelo, en los lugares que manifestaron los valores mínimos y máximos de $\mathrm{pH}$, ECEC, $\mathrm{Al}$ y $\mathrm{Na}$. La relación $\mathrm{Ca} / \mathrm{Mg}$ se mantuvo independiente de las variaciones $\mathrm{pH}$, ECEC y Al, pero sí varió con respecto a la acumulación de Na. Pese a que la disponibilidad de Ca y Mg no era alta, su relación se mantuvo dentro del valor ideal que oscila en- tre 2.5 a 5, lo cual habría sido una ventaja a favor de la producción de cosechas, a pesar de que los agricultores del terreno no realizaban ningún tipo de enmienda con cales agrícolas, dolomitas, ni abonos orgánicos. No obstante, la relación $\mathrm{Ca} / \mathrm{Mg}$ no es suficiente por sí misma para indicar la fertilidad del suelo, ya que la baja disponibilidad de ambos nutrientes es limitada y necesita ser comparada con el K.

La relación $\mathrm{Ca} / \mathrm{K}$ tuvo un detrimento por los valores de bajos de $\mathrm{pH}$, más que por el aumento de $\mathrm{Na}$ y $\mathrm{Al}$. Dado que el rango adecuado de esta relación es de 5 a 25 y todas las relaciones obtenidas en el estudio fueron menores de 8 , se considera que hay muy poca disponibilidad de calcio. Este hallazgo está en concordancia con la relación $(\mathrm{Ca}-\mathrm{Mg}) / \mathrm{K}$, que debió encontrarse entre 10 y 40; pero en el estudio se obtuvo valores inferiores a 6 , demostrando que la causa era la deficiencia de Ca y $\mathrm{Mg}$, y no un aumento de disponibilidad $\mathrm{K}$.

La relación (Ca-Mg)/K mejoraba en las zonas de mayor $\mathrm{pH}$, ECEC y de menor $\mathrm{Al}$ y $\mathrm{Na}$, debido que el $\mathrm{Mg}$ se encontraba menos disponible y no a una abundancia de K; fenómeno que a su vez se repitió con la relación $\mathrm{Mg} / \mathrm{K}$. Esto dejó claro que, para mejorar la fertilidad del suelo se hace necesario realizar enmiendas con cal dolomita para proporcionar a la vez Ca y Mg al suelo.

La relación $\mathrm{Ca} / \mathrm{P}$ fue menor en las zonas con mayor $\mathrm{pH}$, debido a una mayor disponibili- 


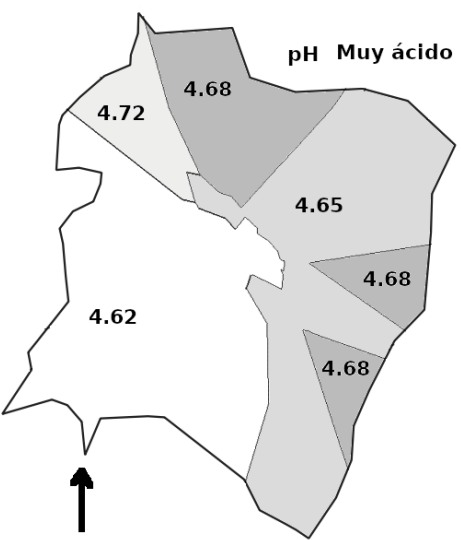

A

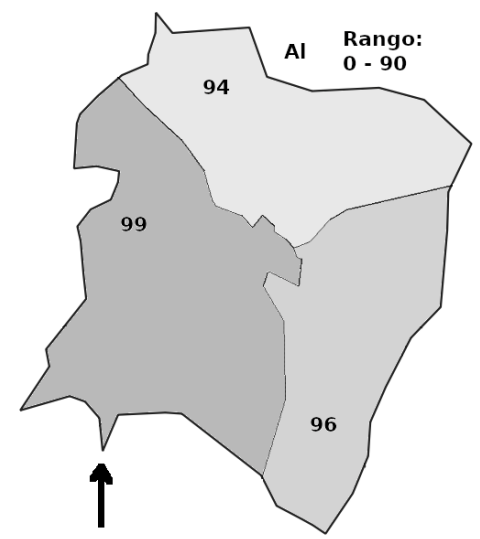

D

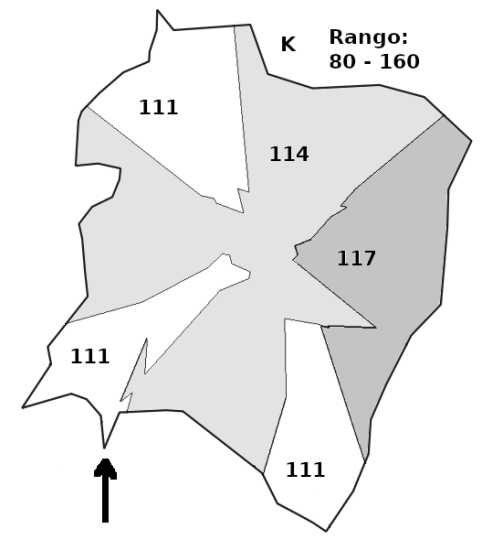

G

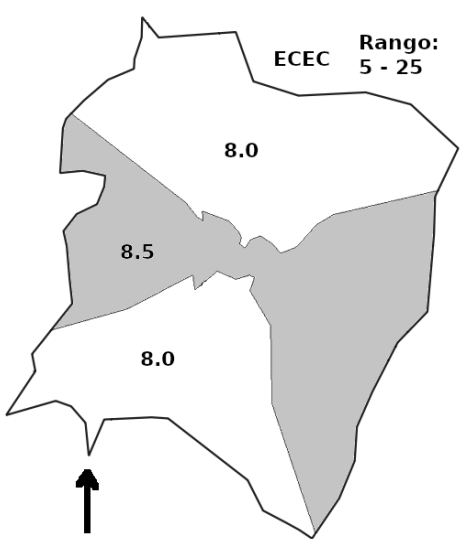

B

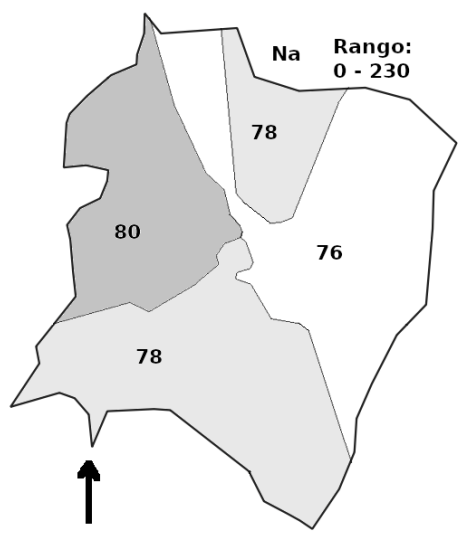

E

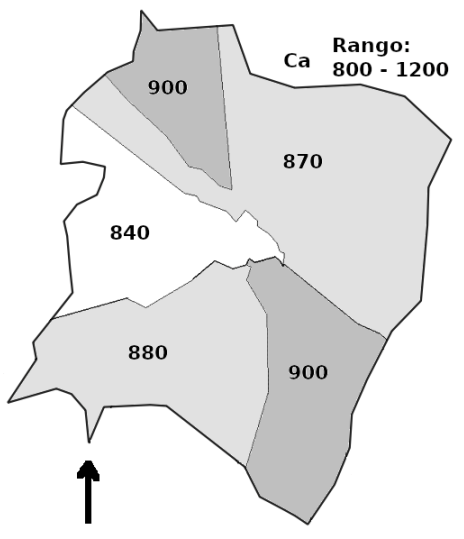

$\mathrm{H}$

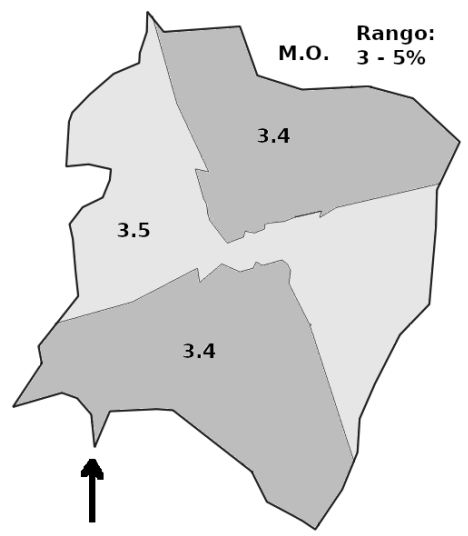

C

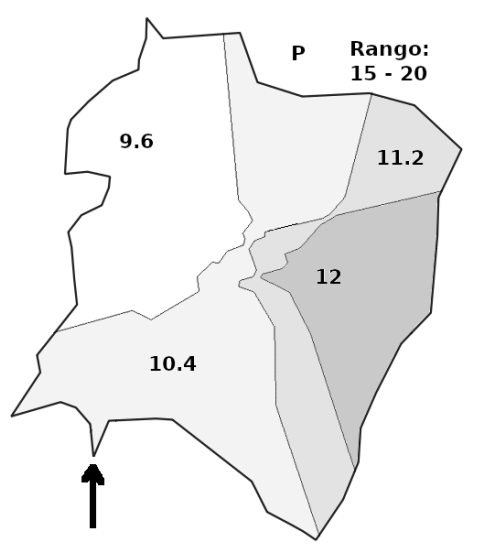

F

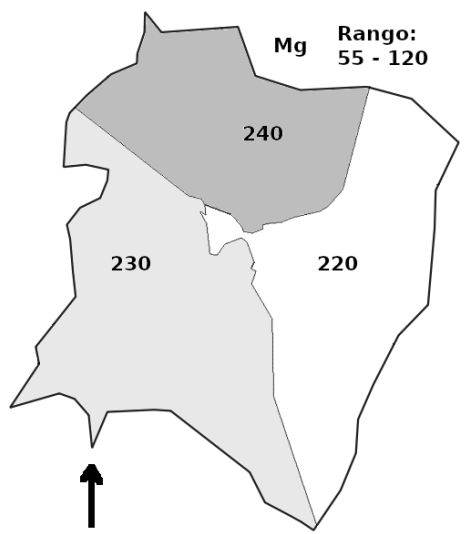

I

Figura 3. Condiciones de variables de fertilidad: (A) pH; (B) ECEC en mEq/100cc; (C) O.M. está en porcentaje. Los antinutrientes (D) Al y (E) Na, al igual que los nutrientes (F) $P,(G) K,(H)$ Ca y (I) Mg, muestran sus datos en PPM. Se detalla la situación o rango ideal para cada variable. 


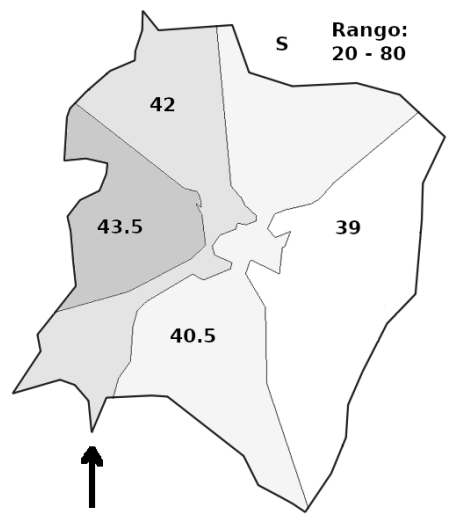

A

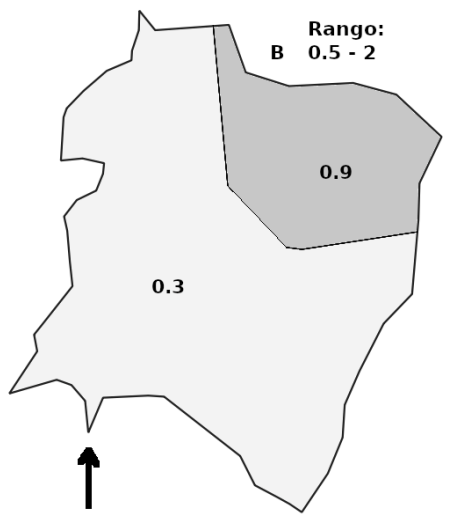

D

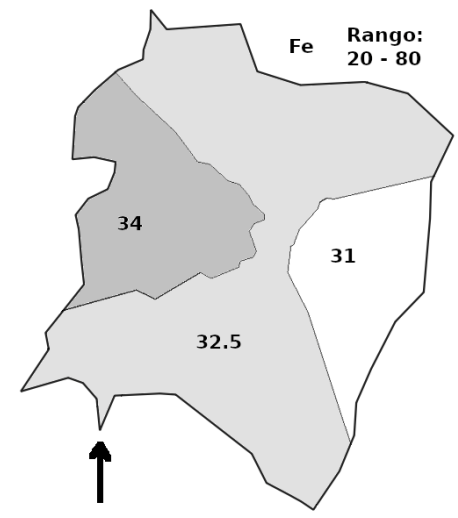

B

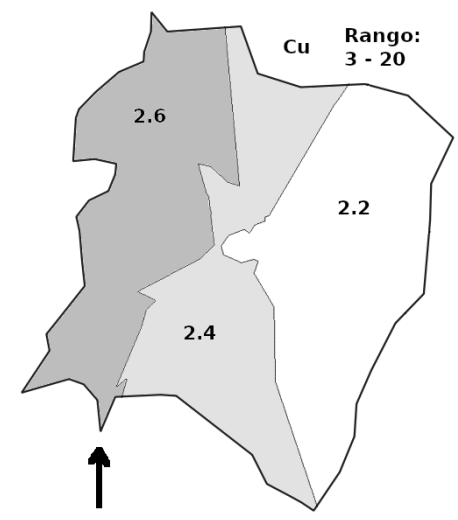

E

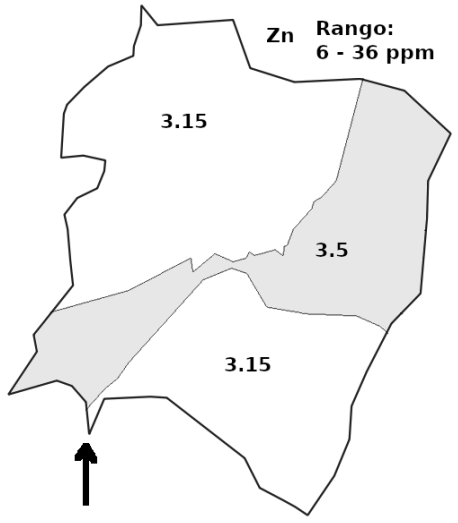

C

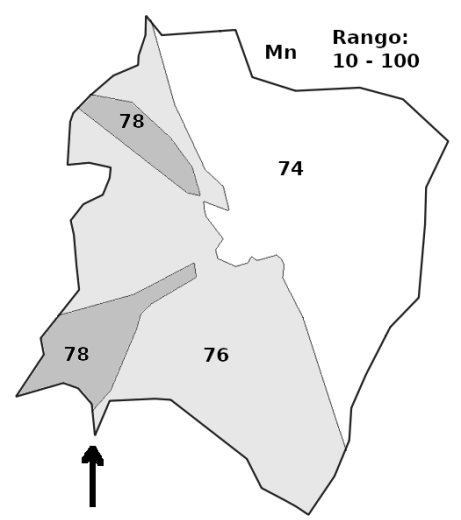

$\mathrm{F}$

Figura 4. Disponibilidad de nutrientes: (A) S, (B) Fe, (C) Zn, (D) B, (E) Cu, (F) Mn. La concentración está en PPM. Se provee el rango ideal para cada variable.

dad de P; mientras que este último nutriente mostró más variabilidad en función de la concentración de $\mathrm{Al}$ y $\mathrm{Na}$. En vista que el calcio es antagonista de la disponibilidad de fósforo, su relación no debería ser mayor a 76, ya que eso conduce a la inmovilización del $\mathrm{P}$, debido a la formación de fosfatos de calcio. Por esta razón se justifica la fertilización fosforada a lo largo de todo el terreno. Es posible suponer que, en el caso de una enmienda caliza, la relación $\mathrm{Ca} / \mathrm{P}$ podría incrementar su valor debido a la inmovilización del $\mathrm{P}$; pero en ese caso, la modificación del pH e inmovilización del Al pueden contribuir a cierta mejora en la solubilidad del P (Penn \& Camberato, 2019).

Por su parte, la relación Fe/P mostró poca variación a los de $\mathrm{pH}$, ECEC y Al; pero su variabilidad aumentaba en función del $\mathrm{Na}$, debido a su conocido antagonismo con $\mathrm{Fe}$, en lugar de que los valores subieran ante una posible mejora en la disponibilidad de P. El rango adecuado de esta relación es de a 1 a 6 , lo cual es una condición intermedia en la cual el Fe 
Tabla 5. Relaciones indicadoras de fertilidad

\begin{tabular}{|l|c|c|c|c|c|c|c|}
\hline Variable & Valor & $\mathbf{C a} / \mathbf{M g}$ & $\mathbf{C a} / \mathbf{K}$ & $\mathbf{( C a}-\mathbf{M g}) / \mathbf{K}$ & $\mathbf{M g} / \mathbf{K}$ & $\mathbf{C a} / \mathbf{P}$ & $\mathbf{F e} / \mathbf{P}$ \\
\hline $\mathrm{pH}$ & 4.62 & 3.7 & 7.6 & 5.6 & 2.0 & 86.0 & 3.3 \\
\hline $\mathrm{pH}$ & 4.72 & 3.7 & 7.9 & 5.8 & 2.2 & 91.7 & 3.4 \\
\hline $\mathrm{ECEC}$ & $8^{(1)}$ & 3.8 & 7.6 & 5.6 & 2.0 & 85.5 & 3.3 \\
\hline $\mathrm{ECEC}$ & 8.5 & 3.8 & 7.4 & 5.4 & 1.9 & 79.2 & 3.1 \\
\hline $\mathrm{Al}$ & $94^{(2)}$ & 3.7 & 7.8 & 5.7 & 2.1 & 88.5 & 3.3 \\
\hline $\mathrm{Al}$ & 99 & 3.7 & 7.6 & 5.6 & 2.0 & 86.0 & 3.4 \\
\hline $\mathrm{Na}$ & $76^{(3)}$ & 4.0 & 7.4 & 5.6 & 1.9 & 72.5 & 2.6 \\
\hline $\mathrm{Na}$ & 80 & 3.7 & 7.4 & 5.4 & 2.0 & 87.5 & 3.5 \\
\hline
\end{tabular}

Nota: ${ }^{(1)} \mathrm{mEq} / 100 \mathrm{cc}^{(2)} \mathrm{y}^{(3)} \mathrm{ppm}$

no causa un antagonismo relevante en la asimilación de $\mathrm{P}$.

Desde el punto de vista agronómico, los resultados demuestran que las prácticas de agricultura de subsistencia aplicadas, través de los años a la producción tradicional de maíz y frijol, han deteriorado la fertilidad del suelo y, por ende, se ha reducido su potencial de cosecha. Estos resultados son consistentes con los encontrados por Haileslassie et al. (2005), quienes reportaron que las prácticas empíricas típicas de los países en vías de desarrollo, son a la larga contraproducentes en términos de su seguridad alimentaria y sostenimiento económico.

El método utilizado en este estudio va más allá de ser una mera herramienta de diagnóstico, sino que puede combinarse con tecnologías de agricultura de precisión para incrementar la eficiencia de uso de fertilizan- tes y otros tratamientos, tanto al suelo como foliares. Esto está en concordancia con lo reportado por Yousefi y Razdari (2015), quienes evidenciaron la nueva tendencia mundial de la integración de diagnósticos modelados por GIS, basados en coordenadas GPS para la creación de bases de datos de suelos, semejantes a las desarrolladas recientemente por el Departamento de Agricultura de los Estados Unidos de América.

Por su parte, Guobin (2011) recomendó aplicar estas tecnologías para el monitoreo intensivo de variables ambientales y de desarrollo de cultivos con el fin de mejorar la producción de alimentos. Sood et al. (2015) indicaron las múltiples aplicaciones de GIS para el mapeo móvil, mitigación de riesgos y respuesta a emergencias. Ye et al. (2013) fueron mucho más lejos al acuñar el término "Geoinformática" como una nueva rama de la ciencia que, 
en asocio con el Internet de las Cosas, permite desarrollar un Sistemas de Manejo por Agricultura de Precisión (PAMS, por sus siglas en inglés), Esto no solo implica el control de aparatos como drones y fertilizadores, sino también que las infraestructuras informáticas deberán ser capaces de apoyar integralmente a los productores de alimentos.

En lo concerniente a la temática de métodos de investigación aplicada, los autores del presente artículo han publicado recientemente un par investigaciones en las que se ha sugerido y fundamentado, suficientemente, el uso de este método para recolectar muestras georeferenciadas de suelo. Todo ello con el objetivo de someterlas a experimentación de laboratorio, introduciendo luego los resultados en una base de datos que permita hacer mapas de interpolación para establecer modelos predictivos de respuesta a diversos tra- tamientos que, respectivamente, podrían manifestarse en un terreno real (Martínez, 2019; Martínez y Somoza, 2020).

\section{Conclusiones}

Con base en los valores calculados de variograma, se generaron mapas de interpolación de Kriging que representan confiablemente la variabilidad espacial de propiedades importantes de la fertilidad de un suelo agrícola. Aunque los métodos estadísticos clásicos no arrojaron información útil para el diagnóstico de condiciones específicas de sitio, fue posible utilizar software GIS para identificar y dividir las zonas cuyos datos tuvieron cuantificación semejante a través de análisis de laboratorio. Este es un método de costo relativamente bajo, pero muy recomendable para la elaboración de planes de manejo agronómico, que permitan incrementar el potencial de cosechas y reducir desperdicios.

\section{Referencias}

Achudume, A. C. (2007). Assessment of farmland sediments after flooding in Ubeji Land in Niger Delta of Nigeria. Environmental monitoring and assessment, 135(1-3), 335-338. Doi: https://doi.org/10.1007/s10661-007-9653

Amaral, L. R. D. y Justina, D. D. D. (2019). Spatial dependence degree and sampling neighborhood influence on interpolation process for fertilizer prescription maps. Engenharia Agrícola, 39(SPE), 85-95. Doi: https://doi.org/10.1590/1809-4430-eng.agric.v39nep85-95/2019.

Bernardi, A. D. C.; Bettiol, G. M.; Ferreira, R. D. P.; Santos, K. E. L.; Rabello, L. M. y Inamasu, R. Y. (2016). Spatial variability of soil properties and yield of a grazed alfalfa pasture in Brazil. Precision agriculture, 17(6), 737-752. Doi: https://doi.org/10.1007/s11119-016-9446-9 
Camana, F.A. y Deutsch, C.V. (2019). The Nugget Effect. J.L. Deutsch (Ed.), Geostatistics Lessons. Recuperado de http://www.geostatisticslessons.com/lessons/nuggeteffect

Ching, J.; Phoon, K. K. y Hu, Y. G. (2009). Efficient evaluation of reliability for slopes with circular slip surfaces using importance sampling. Journal of Geotechnical and Geoenvironmental Engineering, 135(6), 768-777. Recuperado de https://ascelibrary.org/doi/10.1061/\%28ASCE\%29GT.1943-5606.0000035

Cockx, L.; Van Meirvenne, M. y De Vos, B. (2007). Using the EM38DD soil sensor to delineate clay lenses in a sandy forest soil. Science Society of America Journal, 71(4), 1314-1322.

Denton, O. A.; Aduramigba-Modupe, V. O.; Ojo, A. O.; Adeoyolanu, O. D.; Are, K. S.; Adelana, A. O. y Oke, A. O. (2017). Assessment of spatial variability and mapping of soil properties for sustainable agricultural production using geographic information system techniques (GIS). Cogent Food \& Agriculture, 3(1), 1279366. Recuperado de https://www.researchgate.net/publication/312149880_Assessment_of_spatial_variability_and_mapping_of_soil_ properties_for_sustainable_agricultural_production_using_Geographic_Information_ System_Techniques_GIS

Erdogan, E. H.; Erpul, G. y Bayramin, İ. (2007). Use of USLE/GIS methodology for predicting soil loss in a semiarid agricultural watershed. Environmental monitoring and assessment, 131(1-3), 153-161. Doi: https://doi.org/10.1007/s10661-006-9464-6

Farooque, A. A.; Zaman, Q. U.; Schumann, A. W.; Madani, A. y Percival, D. C. (2012). Delineating management zones for site specific fertilization in wild blueberry fields. Applied Engineering in Agriculture. Doi: https:// doi.org/10.13031/2013.41286

Fox, C. A. y Protz, R. (1981). Definition of fabric distributions to characterize the rearrangement of soil particles in the Turbic Cryosols. Canadian Journal of Soil Science, 61(1), 29-38. Doi: https://doi.org/10.4141/cjss81-004

Gia Pham, T.; Kappas, M.; Van Huynh, C. y Hoang Khanh Nguyen, L. (2019). Application of ordinary kriging and regression kriging method for soil properties mapping in hilly region of Central Vietnam. ISPRS International Journal of Geo-Information, 8(3), 147. Doi: https:// doi.org/10.3390/ijgi8030147

Grant, C. A. (2018). Influence of phosphate fertilizer on cadmium in agricultural soils and 
crops. In Phosphate in Soils (pp. 140-165). CRC Press. Recuperado de https://www.researchgate.net/publication/285492498_Influence_of_phosphate_fertilizer_on_cadmium_in_ agricultural_soils_and_crops

Gregorich, E. G. y Carter, M. R. (2007). Soil sampling and methods of analysis. CRC press.

Guobin, S. (2011). Application of GPS and GIS technology in monitoring system of precision agriculture. Hubei Agricultural Sciences, 50(10), 1948-1950. Recuperado de https://www. routledge.com/Soil-Sampling-and-Methods ofAnalysis/CarterGregorich/book

Haileslassie, A.; Priess, J.; Veldkamp, E.; Teketay, D. y Lesschen, J. P. (2005). Assessment of soil nutrient depletion and its spatial variability on smallholders' mixed farming systems in Ethiopia using partial versus full nutrient balances. Agriculture, ecosystems \& environment, 108(1), 1-16. Doi: https://doi.org/10.1016/j.agee.2004.12.010Get

Hendershot, W. H.; Lalande, H. y Duquette, M. (1993). Ion exchange and exchangeable cations. Soil sampling and methods of analysis, 19, 167-176. Recuperado de https://books. google.com.sv/books?hl=es\&lr=\&id=ZTJsbXsikagC\&oi=fnd\&pg=PA197\&dq=Ion+exchange+and+exchangeable+cations. + Soil+sampling + and + methods + of + analysis \&ots $=\mathrm{V}-$ gAvAM5VS-\&sig=2aL9kkgkWp-

Hlaing, K. T.; Haruyama, S. y Aye, M. M. (2008). Using GIS-based distributed soil loss modeling and morphometric analysis to prioritize watershed for soil conservation in Bago river basin of Lower Myanmar. Frontiers of Earth Science in China, 2(4), 465-478. Doi: https:// doi.org/10.1007/s11707-008-0048-3

Houlong, J.; Daibin, W.; Chen, X.; Shuduan, L.; Hongfeng, W.; Chao, Y.; ... y Lina, G. (2016). Comparison of kriging interpolation precision between grid sampling scheme and simple random sampling scheme for precision agriculture. Eurasian Journal of Soil Science, 5(1), p. 62. Doi: https//doi/ 10.18393/ejss.2016.1.062-073

Jafarnejadi, A. R.; Sayyad, G.; Homaee, M. y Davamei, A. H. (2013). Spatial variability of soil total and DTPA-extractable cadmium caused by long-term application of phosphate fertilizers, crop rotation, and soil characteristics. Environmental monitoring and assessment, 185(5), 4087-4096. Doi: https://doi: 10.1007/s10661-012-2851-2.

Kabir, M.; Nur, S.; Chung, S. O.; Jang, B. E.; Kim, Y. J.; Lee, G. J.; ... y Inoue, E. (2019). Variable 
Fertilizer Recommendation for Grass Production by Image-based Growth Status. J. Fac. Agr., Kyushu Univ, 64(1), 145-155. Doi: https://doi.org/10.1016/j.ifacol.2018.08.053Get

Kato, N.; Imori, H. y Owa, N. (1996). The dissolution of calcium silicate slag fertilizers in flooded soil. Japanese Journal of Soil Science and Plant Nutrition (Japan). Recuperado de https://agris.fao.org/agris-search/search.do?recordID=JP1997003913

Liang, B.; Lehmann, J.; Solomon, D.; Kinyangi, J.; Grossman, J.; O’neill, B.; ... y Neves, E. G. (2006). Black carbon increases cation exchange capacity in soils. Soil Science Society of America Journal, 70(5), 1719-1730. Doi: https://doi.org/10.2136/sssaj2005.0383

Martínez, C. (2019). Predictive analysis of pH response to aglime in a Salvadorian field. International Journal of Applied Research, 5(7), 456-460. Recuperado de https://www.allresearchjournal.com/archives $/$ year=2019\&vol=5\&issue $=7 \&$ part $=F \&$ ArticleId $=5992$

Martínez, C. y Somoza, C. (2020). Predictive model of phosphate availability in soil by using GIS software. International Journal of Academic Research and Development, 5(6). Recuperado de https://www.researchgate.net/publication/345656884_Predictive_model_of_ phosphate_availability_in_soil_by_using_QGIS_software

Matos, G. S. B. D.; Fernandes, A. R.; Wadt, P. G. S.; Pina, A. J. D. A.; Franzini, V. I. y Ramos, H. M. N. (2017). The use of DRIS for nutritional diagnosis in oil palm in the state of Pará. Revista Brasileira de Ciência do Solo, 41. Recuperado de https://www.researchgate.net/publication/314205917_The_Use_of_DRIS_for_Nutritional_Diagnosis_in_Oil_Palm_in_ the_State_of_Para

Molin, J. P. y Faulin, G. D. C. (2013). Spatial and temporal variability of soil electrical conductivity related to soil moisture. Scientia Agricola, 70(1), 01-05. Doi: https://doi.org/10.1590/ S0103-90162013000100001

Nawar, S.; Corstanje, R.; Halcro, G.; Mulla, D. y Mouazen, A. M. (2017). Delineation of soil management zones for variable-rate fertilization: A review. In Advances in agronomy (143), 175-245. Academic Press. Recuperado de https://www.researchgate.net/publication/314107017_Delineation_of_Soil_Management_Zones_for_Variable-Rate_Fertilization_A_Review 
Negreiros, J.; Painho, M.; Aguilar, F. y Aguilar, M. (2010). Geographical information systems principles of ordinary kriging interpolator. Journal of Applied Sciences, 10(11), 852-867. Doi: https://doi.org/10.3923/jas.2010.852.867

Panday, D.; Maharjan, B.; Chalise, D.; Shrestha, R. K. y Twanabasu, B. (2018). Digital soil mapping in the Bara district of Nepal using kriging tool in ArcGIS. PloS one, 13(10), Doi: https://doi.org/10.1371/journal.pone.0206350

Pandey, A.; Mathur, A.; Mishra, S. K. y Mal, B. C. (2009). Soil erosion modeling of a Himalayan watershed using RS and GIS. Environmental Earth Sciences, 59(2), 399-410. Doi: https:// doi.org/10.1007/s12665-009-0038-0

Pant, H. K.; Nair, V. D.; Reddy, K. R.; Graetz, D. A. y Villapando, R. R. (2002). Influence of flooding on phosphorus mobility in manure-impacted soil. Journal of environmental quality, 31(4), 1399-1405. Doi: https://doi.org/10.2134/jeq2002.1399

Penn, C. J. y Camberato, J. J. (2019). A critical review on soil chemical processes that control how soil pH affects phosphorus availability to plants. Agriculture, 9(6), p. 120. Doi: https:// doi.org/10.3390/agriculture9060120

Qureshi, A. S.; Hussain, M. I.; Ismail, S. y Khan, Q. M. (2016). Evaluating heavy metal accumulation and potential health risks in vegetables irrigated with treated wastewater. Chemosphere, 163, 54-61. Doi: https://doi.org/10.1016/j.chemosphere.2016.07.073

Rogowski, A. S. (1996). Quantifying soil variability in GIS applications: II Spatial distribution of soil properties. International Journal of Geographical Information Systems, 10(4), 455475. Doi: https://doi.org/10.1080/02693799608902090

Scanlan, C. A.; Brennan, R. F.; D’Antuono, M. F. y Sarre, G. A. (2017). The interaction between soil $\mathrm{pH}$ and phosphorus for wheat yield and the impact of lime-induced changes to soil aluminium and potassium. Soil research, 55(4), 341-353. Doi: https://doi.org/10.1071/ SR16274

Schalscha, E. B.; Pratt, P. F. y Soto, D. (1974). Effect of Phosphate Adsorption on the Cation-Exchange Capacity of Volcanic Ash Soils 1. Soil Science Society of America Journal, 38(3), 539-540. Doi: https://doi.org/10.2136/sssaj1974.03615995003800030044x 
Scull, P.; Franklin, J.; Chadwick, O. A. y McArthur, D. (2003). Predictive soil mapping: a review. Progress in Physical Geography, 27(2), 171-197. Doi: https://doi.org/10.1191/ 0309133303pp366ra

Shi, R.; Li, J.; Jiang, J.; Mehmood, K.; Liu, Y.; Xu, R. y Qian, W. (2017). Characteristics of biomass ashes from different materials and their ameliorative effects on acid soils. Journal of Environmental Sciences, 55, 294-302. Doi: https://doi.org/10.1016/j.jes.2016.07.015

Sood, K.; Singh, S.; Rana, R. S.; Rana, A.; Kalia, V. y Kaushal, A. (2015). Application of GIS in precision agriculture. In Paper presented as lead lecture in national seminar on "Precision farming technologies for high Himalayas, 04-05. Doi: https://doi.org/10.13140/ RG.2.1.2221.3368

Walvoort, D. J.; Brus, D. J. y De Gruijter, J. J. (2010). An R package for spatial coverage sampling and random sampling from compact geographical strata by k-means. Computers \& Geosciences, 36(10), 1261-1267. Doi: https://doi.org/10.1016/j.cageo.2010.04.005

Whelan, B. M.; McBratney, A. B. y Minasny, B. (2002). Vesper 1.5-spatial prediction software for precision agriculture. 6th Int. Conf. on Precision Agriculture, ASA/CSSA/SSSA, Madison, WI, USA (179). Recuperado de http://leg.est.ufpr.br/lib/exe/fetch.php/disciplinas:geoesalq:edercomunello-artigol.pdf

Ye, J.; Chen, B.; Liu, Q. y Fang, Y. (2013, June). A precision agriculture management system based on Internet of Things and WebGIS. In 2013 21st International Conference on Geoinformatics (pp. 1-5). IEEE. Doi: https://doi: 10.1109/Geoinformatics.2013.6626173.

Yousefi, M. R. y Razdari, A. M. (2015). Application of GIS and GPS in precision agriculture (a review). Int. J. Adv. Biol. Biom. Res, 3(1), 7-9. Recuperado de http://www.ijabbr.com/article_12206_798e250c5637a2409e80d6ab4c12f38e.pdf

Zhu, A. X.; Hudson, B.; Burt, J.; Lubich, K. y Simonson, D. (2001). Soil mapping using GIS, expert knowledge, and fuzzy logic. Soil Science Society of America Journal, 65(5), 1463-1472. Doi: https://doi.org/10.2136/sssaj2001.6551463x 